\title{
Inflammatory changes in lung tissues associated with altered inflammation-related microRNA expression after intravenous administration of gold nanoparticles in vivo
}

Cheng-Teng Ng, ${ }^{1}$ Jia'En Jasmine Li, ${ }^{1,2}$ Suresh Kumar Balasubramanian, ${ }^{3}$ Fang You, ${ }^{2}$ Lin-Yue Lanry

Yung, ${ }^{2 *}$ Boon-Huat Bay ${ }^{1 *}$

${ }^{1}$ Department of Anatomy, Yong Loo Lin School of Medicine, National University of Singapore,

Singapore 117597, Singapore

${ }^{2}$ Department of Chemical \& Biomolecular Engineering, Faculty of Engineering, National University of

Singapore, Singapore 117585, Singapore

${ }^{3}$ Department of Civil and Environmental Engineering, National University of Singapore, Singapore

117576

Supporting information

Supporting information is available free of charge online.

Number of pages: 5

Number of figures: 1

Number of tables: 1 


\section{Supplementary information}

Table S1: 119 transcripts predicted to be potential targets for miR-327.

\begin{tabular}{|c|c|c|c|}
\hline $\begin{array}{l}\text { Human } \\
\text { ortholog of }\end{array}$ & $\begin{array}{l}\text { Representative } \\
\text { transcript }\end{array}$ & Gene name & $\begin{array}{l}\text { Total } \\
\text { contex+ }\end{array}$ \\
\hline RBFOX2 & NM_001031695 & RNA binding protein, fox-1 homolog (C. elegans) 2 & -0.61 \\
\hline MPZ & NM_000530 & myelin protein zero & -0.57 \\
\hline ICA1L & NM_138468 & islet cell autoantigen $1,69 \mathrm{kDa}$-like & -0.54 \\
\hline CANX & NM_001024649 & calnexin & -0.52 \\
\hline SYN1 & NM_006950 & synapsin I & -0.52 \\
\hline FAM59B & NM_001168241 & family with sequence similarity 59 , member B & -0.49 \\
\hline C20orf203 & NM_182584 & chromosome 20 open reading frame 203 & -0.48 \\
\hline SLITRK4 & NM_001184749 & SLIT and NTRK-like family, member 4 & -0.46 \\
\hline SOX6 & NM_001145811 & SRY (sex determining region Y)-box 6 & -0.45 \\
\hline ATXN7L2 & NM_153340 & ataxin 7-like 2 & -0.43 \\
\hline PDCD1LG2 & NM_025239 & programmed cell death 1 ligand 2 & -0.42 \\
\hline ONECUT1 & NM_004498 & one cut homeobox 1 & -0.4 \\
\hline TNRC6A & NM_014494 & trinucleotide repeat containing $6 \mathrm{~A}$ & -0.39 \\
\hline NRBF2 & NM_030759 & nuclear receptor binding factor 2 & -0.39 \\
\hline AKIRIN1 & NM_001136275 & akirin 1 & -0.36 \\
\hline CPEB2 & NM_001177381 & cytoplasmic polyadenylation element binding protein 2 & -0.35 \\
\hline ARHGAP36 & NM_144967 & Rho GTPase activating protein 36 & -0.35 \\
\hline MED20 & NM_004275 & mediator complex subunit 20 & -0.34 \\
\hline CREB1 & NM_004379 & cAMP responsive element binding protein 1 & -0.33 \\
\hline PREP & NM_002726 & prolyl endopeptidase & -0.33 \\
\hline CAMK2N1 & NM_018584 & calcium/calmodulin-dependent protein kinase II inhibitor 1 & -0.32 \\
\hline FAM189A1 & NM_015307 & family with sequence similarity 189 , member A1 & -0.31 \\
\hline THRAP3 & NM_005119 & thyroid hormone receptor associated protein 3 & -0.31 \\
\hline KIF14 & NM_014875 & kinesin family member 14 & -0.3 \\
\hline ZNF827 & NM_178835 & zinc finger protein 827 & -0.29 \\
\hline CCDC41 & NM_001042399 & coiled-coil domain containing 41 & -0.29 \\
\hline GMPPB & NM_013334 & GDP-mannose pyrophosphorylase B & -0.28 \\
\hline UBR1 & NM_174916 & ubiquitin protein ligase $\mathrm{E} 3$ component $\mathrm{n}$-recognin 1 & -0.28 \\
\hline PTPRC & NM_002838 & protein tyrosine phosphatase, receptor type, $\mathrm{C}$ & -0.27 \\
\hline IL1A & NM_000575 & interleukin 1, alpha & -0.27 \\
\hline RUNX1T1 & NM_001198625 & runt-related transcription factor 1 ; translocated to, 1 (cyclin D-related) & -0.26 \\
\hline PTPRD & NM_001040712 & protein tyrosine phosphatase, receptor type, $\mathrm{D}$ & -0.26 \\
\hline ACSL3 & NM_004457 & acyl-CoA synthetase long-chain family member 3 & -0.26 \\
\hline MRVI1 & NM_001098579 & murine retrovirus integration site 1 homolog & -0.25 \\
\hline NAMPT & NM_005746 & nicotinamide phosphoribosyltransferase & -0.25 \\
\hline ARID4B & NM_001206794 & $\mathrm{AT}$ rich interactive domain $4 \mathrm{~B}$ (RBP1-like) & -0.25 \\
\hline CASC3 & NM_007359 & cancer susceptibility candidate 3 & -0.24 \\
\hline
\end{tabular}




\begin{tabular}{|c|c|c|c|}
\hline UBXN4 & NM_014607 & UBX domain protein 4 & -0.24 \\
\hline C6orf223 & NM_001171992 & chromosome 6 open reading frame 223 & -0.24 \\
\hline TFAP2A & NM_001032280 & transcription factor AP-2 alpha (activating enhancer binding protein 2 alpha) & -0.24 \\
\hline SLC16A2 & NM_006517 & solute carrier family 16, member 2 (monocarboxylic acid transporter 8 ) & -0.23 \\
\hline BAI3 & NM_001704 & brain-specific angiogenesis inhibitor 3 & -0.23 \\
\hline ARL5A & NM_001037174 & ADP-ribosylation factor-like $5 \mathrm{~A}$ & -0.22 \\
\hline SIAH3 & NM_198849 & seven in absentia homolog 3 (Drosophila) & -0.22 \\
\hline TSPYL5 & NM_033512 & TSPY-like 5 & -0.22 \\
\hline DPYSL3 & NM_001197294 & dihydropyrimidinase-like 3 & -0.22 \\
\hline WSB1 & NM_015626 & WD repeat and SOCS box containing 1 & -0.21 \\
\hline $\mathrm{EIF} 2 \mathrm{C} 3$ & NM_024852 & eukaryotic translation initiation factor $2 \mathrm{C}, 3$ & -0.21 \\
\hline TFAP2B & NM_003221 & transcription factor AP-2 beta (activating enhancer binding protein 2 beta) & -0.21 \\
\hline INHBE & NM_031479 & inhibin, beta $\mathrm{E}$ & -0.21 \\
\hline PLEKHG6 & NM_001144856 & pleckstrin homology domain containing, family $\mathrm{G}$ (with RhoGef domain) member 6 & -0.2 \\
\hline ERCC4 & NM_005236 & excision repair cross-complementing rodent repair deficiency, complementation group & -0.19 \\
\hline SMARCE1 & NM_003079 & SWI/SNF related, matrix associated, actin dependent regulator of chromatin, & -0.18 \\
\hline RTN3 & NM_006054 & reticulon 3 & -0.18 \\
\hline FOXP4 & NM_001012426 & forkhead box P4 & -0.18 \\
\hline MAPK6 & NM_002748 & mitogen-activated protein kinase 6 & -0.17 \\
\hline KLLN & NM_001126049 & killin, p53-regulated DNA replication inhibitor & -0.17 \\
\hline IGDCC3 & NM_004884 & immunoglobulin superfamily, DCC subclass, member 3 & -0.17 \\
\hline PCYT1B & NM_001163264 & phosphate cytidylyltransferase 1 , choline, beta & -0.17 \\
\hline $\mathrm{KCNH} 2$ & NM_000238 & potassium voltage-gated channel, subfamily $\mathrm{H}$ (eag-related), member 2 & -0.17 \\
\hline SLC9A9 & NM_173653 & solute carrier family 9 (sodium/hydrogen exchanger), member 9 & -0.17 \\
\hline TRIM66 & NM_014818 & tripartite motif containing 66 & -0.17 \\
\hline RYR1 & NM_000540 & ryanodine receptor 1 (skeletal) & -0.16 \\
\hline CACNA1D & NM_000720 & calcium channel, voltage-dependent, L type, alpha 1D subunit & -0.16 \\
\hline PHC3 & NM_024947 & polyhomeotic homolog 3 (Drosophila) & -0.15 \\
\hline WTIP & NM_001080436 & Wilms tumor 1 interacting protein & -0.15 \\
\hline RIMBP3 & NM_015672 & RIMS binding protein 3 & -0.15 \\
\hline TRIM44 & NM_017583 & tripartite motif containing 44 & -0.15 \\
\hline STRBP & NM_001171137 & spermatid perinuclear RNA binding protein & -0.15 \\
\hline C3orf63 & NM_001112736 & chromosome 3 open reading frame 63 & -0.14 \\
\hline CPEB4 & NM_030627 & cytoplasmic polyadenylation element binding protein 4 & -0.14 \\
\hline C14orf119 & NM_017924 & chromosome 14 open reading frame 119 & -0.14 \\
\hline AFAP1L1 & NM_001146337 & actin filament associated protein 1-like 1 & -0.14 \\
\hline NEDD4 & NM_006154 & neural precursor cell expressed, developmentally down-regulated 4 & -0.13 \\
\hline CD274 & NM_014143 & CD274 molecule & -0.13 \\
\hline U2SURP & NM_001080415 & U2 snRNP-associated SURP domain containing & -0.13 \\
\hline ARHGAP35 & NM_004491 & Rho GTPase activating protein 35 & -0.12 \\
\hline GRM5 & NM_000842 & glutamate receptor, metabotropic 5 & -0.12 \\
\hline FKBP4 & NM_002014 & FK506 binding protein $4,59 \mathrm{kDa}$ & -0.12 \\
\hline THRB & NM_000461 & thyroid hormone receptor, beta (erythroblastic leukemia viral (v-erb-a) oncogene & -0.12 \\
\hline APCDD1L & NM_153360 & adenomatosis polyposis coli down-regulated 1-like & -0.12 \\
\hline DLC1 & NM_001164271 & deleted in liver cancer 1 & -0.11 \\
\hline
\end{tabular}




\begin{tabular}{|c|c|c|c|}
\hline TXNRD1 & NM_001093771 & thioredoxin reductase 1 & -0.11 \\
\hline HSD11B2 & NM_000196 & hydroxysteroid (11-beta) dehydrogenase 2 & -0.11 \\
\hline PDIA6 & NM_005742 & protein disulfide isomerase family $\mathrm{A}$, member 6 & -0.11 \\
\hline AP3M1 & NM_012095 & adaptor-related protein complex 3 , mu 1 subunit & -0.11 \\
\hline PRR13 & NM_001005354 & proline rich 13 & -0.1 \\
\hline JPH4 & NM_001146028 & junctophilin 4 & -0.1 \\
\hline VGLL3 & NM_016206 & vestigial like 3 (Drosophila) & -0.1 \\
\hline SMG5 & NM_015327 & smg-5 homolog, nonsense mediated mRNA decay factor (C. elegans) & -0.1 \\
\hline RAB1A & NM_004161 & RAB1A, member RAS oncogene family & -0.1 \\
\hline PPP3R1 & NM_000945 & protein phosphatase 3 , regulatory subunit $\mathrm{B}$, alpha & -0.09 \\
\hline UBE2Q1 & NM_017582 & ubiquitin-conjugating enzyme E2Q family member 1 & -0.09 \\
\hline CCNT2 & NM_001241 & cyclin $\mathrm{T} 2$ & -0.09 \\
\hline PSME3 & NM_005789 & proteasome (prosome, macropain) activator subunit 3 (PA28 gamma; Ki) & -0.09 \\
\hline ZFP91 & NM_001197051 & zinc finger protein 91 homolog (mouse) & -0.09 \\
\hline THAP2 & NM_031435 & THAP domain containing, apoptosis associated protein 2 & -0.08 \\
\hline RGS12 & NM_002926 & regulator of G-protein signaling 12 & -0.07 \\
\hline TIA1 & NM_022037 & TIA1 cytotoxic granule-associated RNA binding protein & -0.07 \\
\hline CDK6 & NM_001145306 & cyclin-dependent kinase 6 & $>-0.07$ \\
\hline TLL2 & NM_012465 & tolloid-like 2 & -0.07 \\
\hline CHTF8 & NM_001039690 & CTF8, chromosome transmission fidelity factor 8 homolog (S. cerevisiae) & -0.06 \\
\hline FAM107A & NM_001076778 & family with sequence similarity 107 , member A & -0.06 \\
\hline C22orf46 & NM_001142964 & chromosome 22 open reading frame 46 & -0.06 \\
\hline CNOT6L & NM_144571 & CCR4-NOT transcription complex, subunit 6-like & -0.05 \\
\hline PDK3 & NM_001142386 & pyruvate dehydrogenase kinase, isozyme 3 & -0.05 \\
\hline SP1 & NM_003109 & Sp1 transcription factor & $>-0.04$ \\
\hline ELAVL1 & NM_001419 & ELAV (embryonic lethal, abnormal vision, Drosophila)-like 1 (Hu antigen R) & -0.04 \\
\hline IGF2BP1 & NM_001160423 & insulin-like growth factor 2 mRNA binding protein 1 & $>-0.02$ \\
\hline KLHL9 & NM_018847 & kelch-like 9 (Drosophila) & $>-0.02$ \\
\hline SPRY4 & NM_001127496 & sprouty homolog 4 (Drosophila) & $>-0.02$ \\
\hline CACNA1C & NM_000719 & calcium channel, voltage-dependent, $\mathrm{L}$ type, alpha $1 \mathrm{C}$ subunit & $>-0.01$ \\
\hline FRMPD4 & NM_014728 & FERM and PDZ domain containing 4 & $>-0.01$ \\
\hline TANC2 & NM_025185 & tetratricopeptide repeat, ankyrin repeat and coiled-coil containing 2 & $>-0.01$ \\
\hline SNRPD1 & NM_006938 & small nuclear ribonucleoprotein $\mathrm{D} 1$ polypeptide $16 \mathrm{kDa}$ & N/A \\
\hline PSMF1 & NM_006814 & proteasome (prosome, macropain) inhibitor subunit 1 (PI31) & N/A \\
\hline AGXT2 & NM_031900 & alanine--glyoxylate aminotransferase 2 & N/A \\
\hline SLC38A9 & NM_173514 & solute carrier family 38 , member 9 & $\mathrm{~N} / \mathrm{A}$ \\
\hline SLC37A2 & NM_198277 & solute carrier family 37 (glycerol-3-phosphate transporter) member 2 & $\mathrm{~N} / \mathrm{A}$ \\
\hline
\end{tabular}




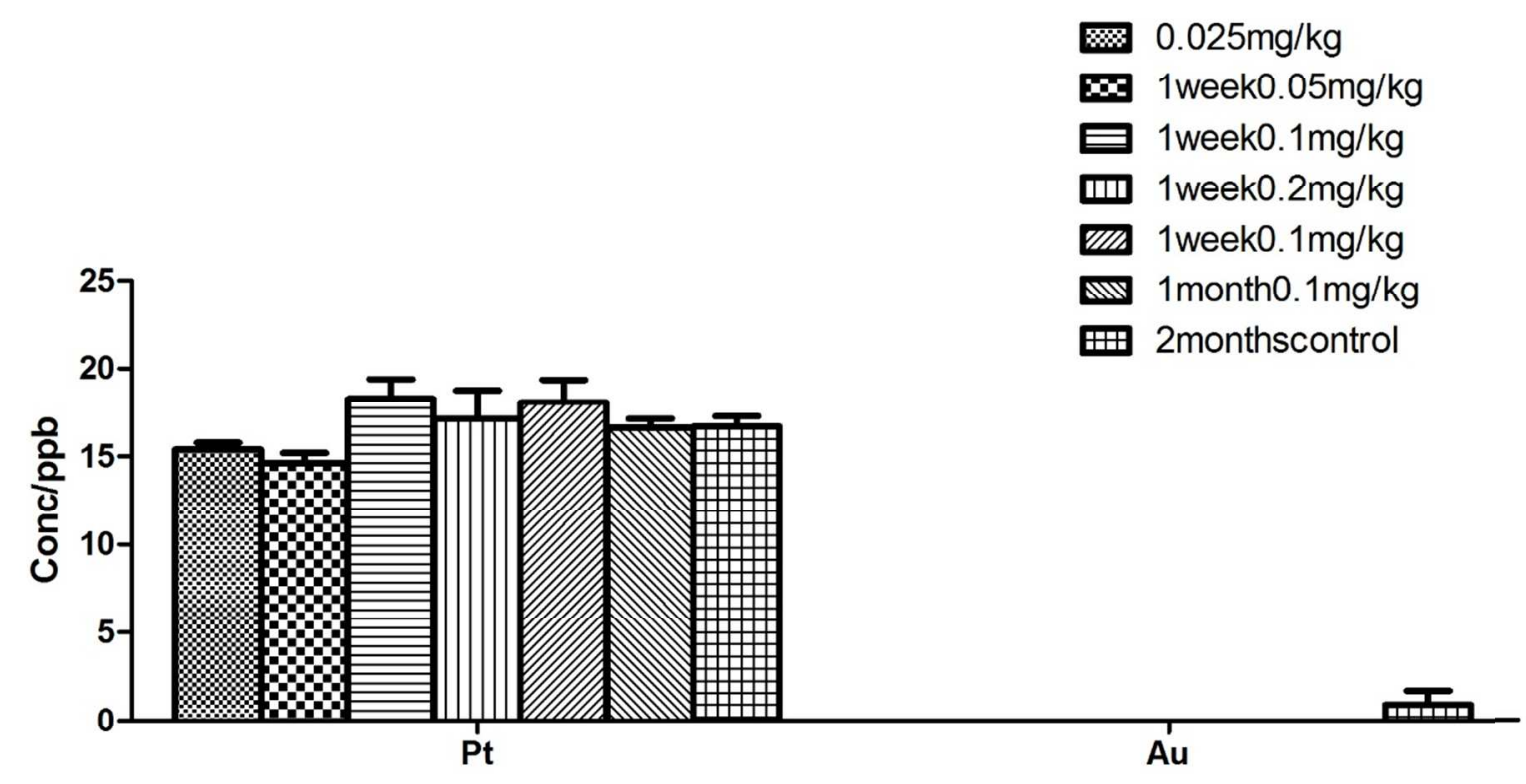

Figure S1: Bar chart showed the absence of $\mathrm{Au}$ in the blood of rat; Platinum (Pt) was used as an internal control. $\mathrm{n}=5$; Error bars $=$ SEM 